\title{
The Measurement of Solvation Forces with Application to Biological Systems
}

\author{
M. J. Higgins, ${ }^{*}$ T. Uchihashi,* M. Polcik, * J. E. Sader,** S.P. Jarvis* \\ *Centre for Research on Adaptive Nanostructures and Nanodevices, University of Dublin, Trinity \\ College, Dublin 2, Ireland \\ **Department of Mathematics and Statistics, University of Melbourne, Victoria 3010, Australia
}

Directly measuring forces in aqueous environments with a mechanical probe of lateral dimensions comparable to that of a single molecule provides an invaluable insight into the processes controlling if and how a molecule approaches another molecule or a membrane. In the immediate vicinity of the molecule, continuum models break down and the aqueous environment will often form a discrete layered structure depending on the nature of the molecule. The absence or presence of such structure may be fundamental in influencing the promotion or inhibition of protein adsorption, biological function and membrane recognition.

In order to perform such measurements it has been necessary to combine a number of innovative techniques with a standard atomic force microscopy (AFM). For high-resolution imaging we use the highly sensitive frequency modulation detection scheme (FM-AFM) in liquid [1, 2] and have subsequently used the method of Sader and Jarvis to extract quantitative force values from frequency shift measurements [3]. To implement FM-AFM successfully in liquid we have used magnetically activated dynamic mode (MAD-mode!) where a small magnetic particle is attached to the end of the cantilever (Fig. 1) and an external magnetic field applied via a current carrying coil. To increase the sensitivity of the measurement to the interaction local to the tip apex we have used a high aspect ratio multiwalled carbon nanotube probe (Fig. 2). This reduces the hydrodynamic squeeze damping between the surface and the bulk of the tip. The nanotube is attached in a specially designed field emission scanning electron microscope, which permits us some control over both the length and direction of the probe.

We have directly measured localised density changes in ultra-pure water at small tip-sample separations and have combined this with nanometer resolution images of the surface. Careful characterisation of the nanotube probe using electron microscopy after the measurement has allowed us to normalise interaction forces as a function of probe radius [4]. Using self-assembled monolayers with varying end groups, we have been able to investigate the role of local surface chemistry and morphology on measured water structure and tip-surface interaction. This has been extended to observations of quantified differences in localised structures in the presence of ions with the structures being dependent on the choice of ion. Further, we have investigated whether oscillatory forces corresponding to water structure are present at the surface of lipid bilayers (Fig.3). We aim to understand the effects of various physiological conditions on water structure, the relevant length scales over which hydration forces interact and the influence of water structure on biological processes.

\section{References}

[1] S. P. Jarvis et al., J. Phys. Chem. B 104 (2000) 6091.

[2] S. P. Jarvis et al., Applied Physics A 72 (2001) S129. 
[3] J. E. Sader and S. P. Jarvis, Appl. Phys. Lett. 84 (2004) 1801.

[4] T. Uchihashi, M.J. Higgins, Y. Nakayama, J.E. Sader and S.P. Jarvis, Nanotechnology 16 (2005) S40.

[5] This research was supported by Science Foundation Ireland Research Grant (01/PI.2/C033) and the Human Frontier Science Program.

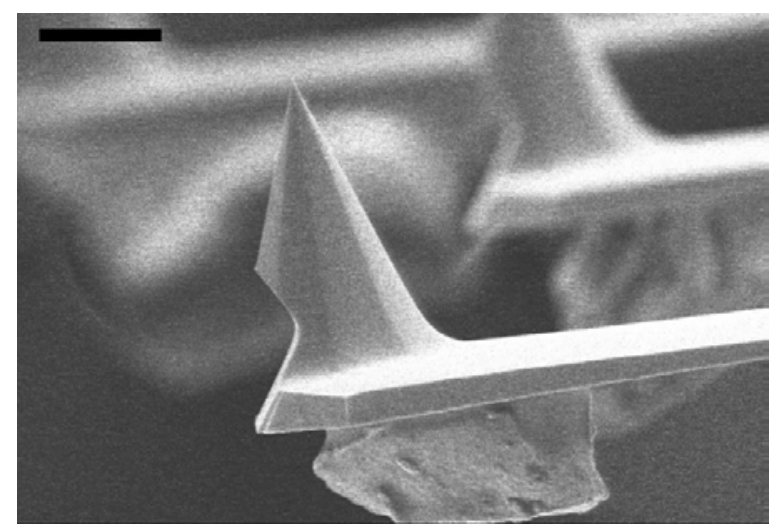

Fig. 1 AFM lever showing glass encapsulated NdBFe magnetic particle. (source Prof. Gil lee, Purdue University, USA). Scale bar is $20 \mu \mathrm{m}$.

Fig. 2 AFM tip with multiwalled carbon nanotube attached.
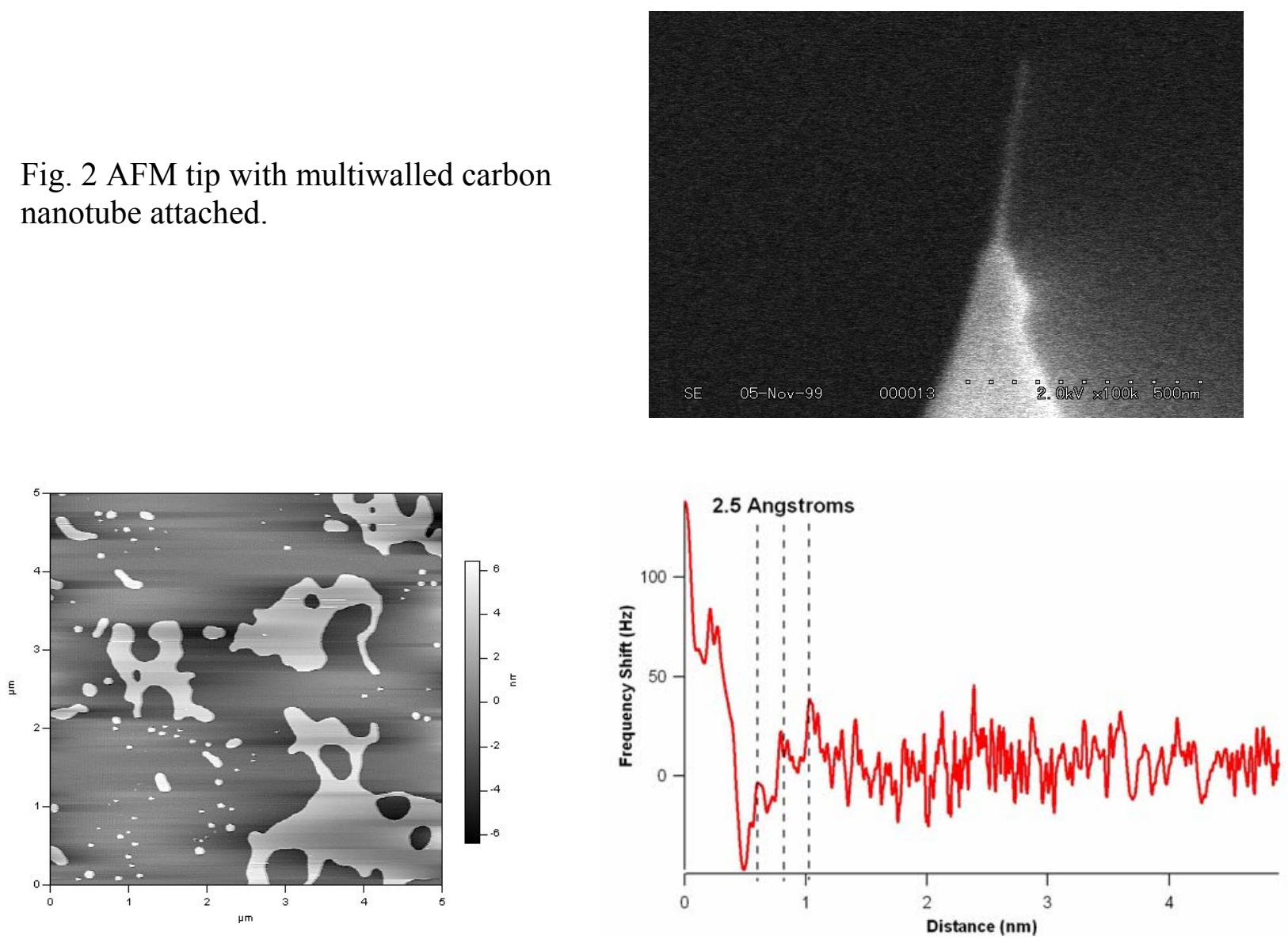

Fig. 3 (a) AFM image of lipid bilayer. (b) Oscillatory solvation force observed on a lipid bilayer. 